\title{
Effects of dynamic taping on shoulder joint proprioception
}

\author{
So-Young Park ${ }^{\mathrm{a}(i)}$, Min-Ji Kim ${ }^{\mathrm{a}(i)}$, So-Eun Seol ${ }^{\mathrm{a}(i)}$, Chan Hwang ${ }^{\mathrm{a}(i)}$, \\ Ji-Su Hong ${ }^{a}{ }^{(i)}$, Ho Kim ${ }^{b \oplus}$, Won-Seob Shin ${ }^{a, b}$ \\ ${ }^{a}$ Department of Physical Therapy, College of Health and Medical Science, Daejeon University, Daejeon, Republic of Korea \\ ${ }^{b}$ Department of Physical Therapy, Graduate School, Daejeon University, Daejeon, Republic of Korea
}

Objective: There are many cases of applying various taping methods to prevent muscle damage and to assist with movement. The purpose of this study was to investigate and to compare the effects of dynamic taping on joint position sense and to find out the difference in error values during various degrees of shoulder flexion.

Design: Cross-sectional study.

Methods: A total of 20 subjects participated in this study with a randomized cross-over design. The order of taping was adjusted by randomly proceeding with dynamic taping, sham taping, and no taping. After the taping, the proprioception of the shoulder joint was evaluated. The evaluation of proprioceptive sensation was performed by evaluating joint position sensation. The sequence was adjusted by randomly performing joint position tests at each shoulder flexion of 50, 90, and 110 degrees. All angles were repeated 3 times.

Results: There was a significant difference between dynamic taping and no taping in 50 degrees of shoulder flexion. There was a significant difference between sham taping and no taping in 90 degrees of shoulder flexion $(p<0.05)$. No significant difference was found in 110 degrees of shoulder flexion.

Conclusions: In this study, it was confirmed that dynamic taping is effective in improving the joint position sense in 50 degrees of shoulder flexion. In the future, it is expected that further studies will be conducted on patients with shoulder dysfunction with decreased proprioception.

Key Words: Motion, Muscles, Proprioception, Shoulder

\section{Introduction}

Proprioception consists of a sensation that recognizes joint movement and detects the position of a joint [1]. This proprioception information is mainly transmitted to the central nervous system through sensory receptors in the skin, muscle, tendon and synovial membranes, and then contributes to functional stability of the joint by appropriately changing the joint position through the reflex and conscious action of the skeletal muscle through efferent fibers [2].

Deficiencies in proprioception can lead to hypermobility of the shoulder complex, an increased risk of a shoulder injury, and dysfunction [3]. Patients with shoulder instability or shoulder dislocation showed that proprioception was significantly lower than those of healthy people, and those who performed movements with the arm above the shoulder frequently put a lot of stress onto the shoulder joint, increasing the risk of shoulder injury [4,5].

To improve proprioception, use of elastic bands and taping have been studied previously, and therapeutic tapes can be categorized into non-elastic tape and elastic tape according to their elasticity [6,7]. Currently, the tapes used for treatment are the McConnell tape (sham tape) for the non-elastic tape and the kinesiology tape and dynamic tape for the elastic tape [8]. Kinesiology tape has an elongation of $140 \%-180 \%$ and does not stretch at the endpoint but stretch-

Received: 30 November, 2020 Revised: 21 December, 2020 Accepted: 21 December, 2020

Corresponding author: Won-Seob Shin (ORCID https://orcid.org/0000-0002-6515-7020)

Department of Physical Therapy, College of Health and Medical Science, Daejeon University, 62 Daehak-ro, Dong-gu, Daejeon 34520, Republic of Korea Tel: 82-42-280-2290 Fax: 82-42-280-2295 E-mail: shinws@dju.kr

(c) This is an Open-Access article distributed under the terms of the Creative Commons Attribution Non-Commercial License (http://creativecommons.org/licenses/ by-nc/4.0) which permits unrestricted non-commercial use, distribution, and reproduction in any medium, provided the original work is properly cited.

Copyright $@ 2020$ Korean Academy of Physical Therapy Rehabilitation Science 
es only in the vertical direction [9]. In addition, since it is attached according to the alignment of the muscle fibers, the space between the muscle and the skin increases, increasing the circulation of blood and lymph, thereby improving the motor function of the muscle [10]. The dynamic tape has more than $200 \%$ elongation and has a resistance and rebound of 10 to $15 \mathrm{~kg}$. It can be stretched not only in the vertical direction but also in the horizontal direction, and as opposed to the kinesiology tape it is applied in a close distance of the body part to which the tape is applied in order to maximize the load absorption and to apply with the tape stretched. From a functional point of view, pre-stretching when attaching the dynamic tape makes it ready to provide support immediately when the muscle and tendon units begin to stretch [11].

Many studies have reported that the elasticity of kinesiology tape enhances muscle strength, range of motion, and proprioception [12]. According to previous studies, dynamic tape has an effect on the provision of external force and proprioception input [11]. However, there is still insufficient research on the influence of dynamic taping on proprioception sense.

Therefore, this study was conducted to find out how dynamic tape affects the joint position sense during shoulder flexion, and various methods of taping are applied to find out the difference in error values during shoulder flexion.

\section{Methods}

\section{Participants}

This study was conducted with 20 healthy adult males and females attending Daejeon University. The criteria for selecting subjects were as follows: 1) those who did not have discomfort in shoulder movement, 2) those who did not have an allergic reaction to taping, and 3 ) those who could perform shoulder flexion movement normally. The exclusion criteria were as follows: 1) those who were diagnosed with orthopedic, neurological, cardiopulmonary function or psychological disease within the last 3 months and 2) those who had shoulder pain or surgery in the last 2 years [13]. Information about the purpose and method of participation in the experiment were explained to the subjects, and after obtaining consent, the subjects were selected. All measurements were performed in 3 sessions that took approximately 30 minutes in a quiet room. This study was approved by the Institutional Review Board of Daejeon University (1040647-202012- HR-006-03).

\section{Procedure}

A total of 20 subjects participated in this study with a randomized cross-over design. The order of taping was adjusted by randomly proceeding with dynamic taping, sham taping, and no taping. After the taping was attached, the order was adjusted by randomly performing joint position sense of 50, 90, and 110 degrees of flexion range of motion of each shoulder. All measurements were repeated three times (Figure 1).

\section{Intervention}

\section{Dynamic taping}

The length of the tape was varied in consideration of the physical differences between sex, and each were attached to 2 places to provide excellent directional vector and to resist the forward movement of the humeral head. Both ends of the tape were rounded with $25 \mathrm{~cm}$ in length for female and $30 \mathrm{~cm}$ in length for male. After assuming a posture with the subject's arm extended forward, a 5-cm strip of tape was applied to $2 / 3$ part of the biceps brachii. After that, with the scapula raised, the tape was applied past the humerus, covered the clavicle, and was stretched by $150 \%$ to the scapular body. The end of the tape was attached without stretching. After establishing a posture with the subject's arm extended backward, a 5 -cm strip of tape was applied to $2 / 3$ of the triceps brachii. Then, with the shoulders raised, the tape was stretched by $150 \%$ to the position of the clavicle through the spine of the scapula. The end of the tape was attached without stretching (Figure 2).

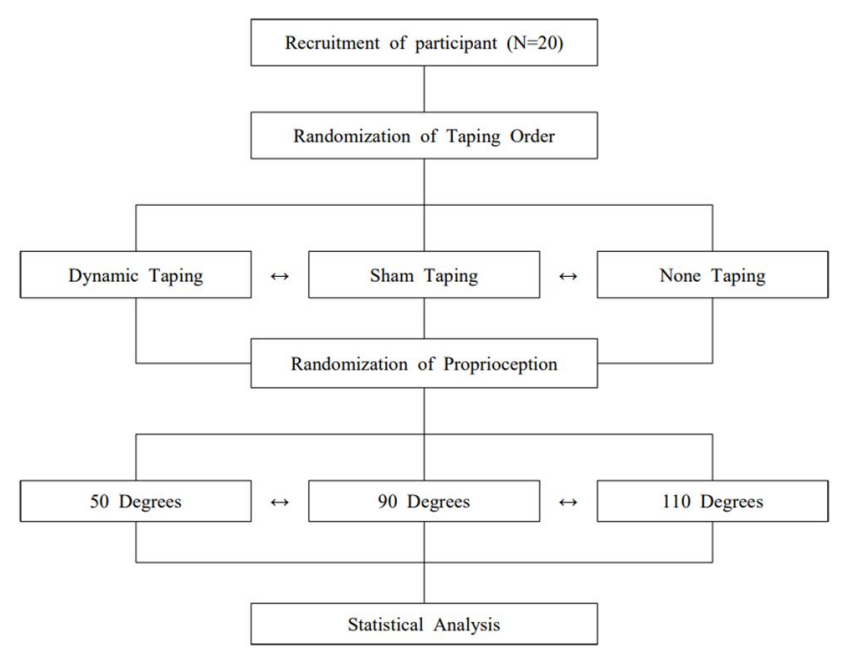

Figure 1. Procedure. 


\section{Sham taping}

The sham tape was attached without tension to the distal part of the deltoid on the side and above the clavicle in the sagittal plane. Sham taping consisted of two $10 \mathrm{~cm}$ I-strips applied without tension using a dynamic tape [14] (Figure 2).

\section{Outcome measures}

\section{Proprioception (joint position sense)}

For the measurement of joint position sense of shoulder flexion, a smartphone app (Clinometer + bubble level; Plaincode $^{\mathrm{TM}}$, Rosenheim, Germany) was used. The subject was blindfolded while sitting on a chair without a backrest and leaning at the corners of the columns from the vertebrae to the head. The subject's humerus central line mark was set, and in a position of 35 degrees in front of the frontal plane, the researcher manually raised the arm to the specified angle $(50,90,110$ degrees $)$ and held it for 5 seconds. After that, the researcher returned the subject's arm in the starting position and then allowed them to raise the arm at the same specified

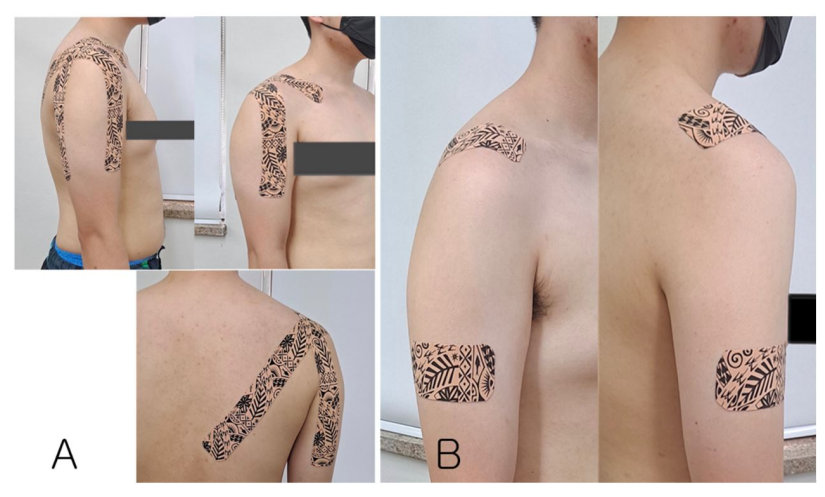

Figure 2. Taping application methods. (A) Dynamic taping. (B) Sham taping.

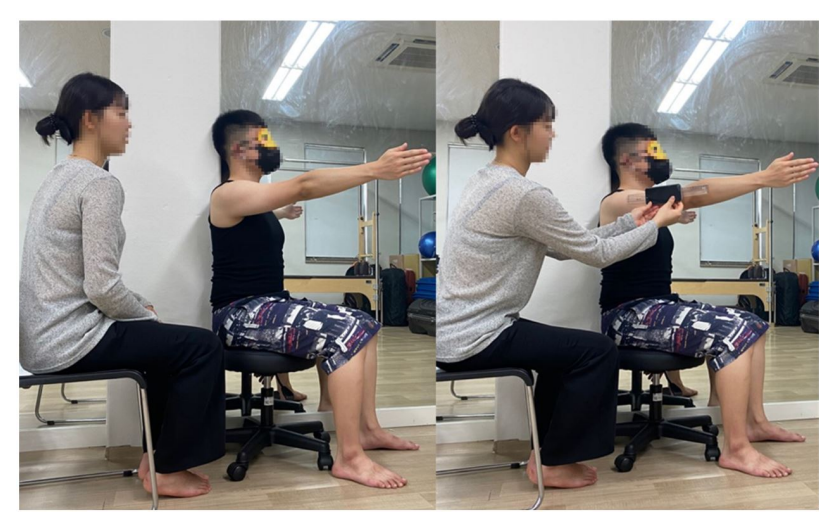

Figure 3. Proprioception (joint position sense test). angle. At this time, errors in proprioception was prevented by keeping the subject's body from touching the researcher's body [15] (Figure 3).

\section{Data and statistical analysis}

SPSS for Windows, Version 25.0 (IBM Co., Armonk, NY, USA) was used for the statistical analysis of the collected data. For general characteristics of the subjects, the mean and standard deviation were presented using descriptive statistics. One-way ANOVA with repeated measure was performed to analyze the differences in angles while using same tape application method and to analyze by the tape types at the same angle. Bonferroni correction was performed as a post-analysis, and the statistical significance level was set to $\alpha=0.05$.

\section{Results}

With 12 subjects being male and 8 subjects being female, a total of 20 subjects participated in this study. The general characteristics of this study are shown in the following table (Table 1).

At 50 degrees, there was a significant difference between the methods in the comparison according to each condition $(p<0.05)$. As a result of post-hoc analysis, the angular error value of the dynamic taping was significantly smaller than that of the sham taping and the no taping conditions $(p<0.05)$. There was a significant difference between the methods in the comparison according to each condition at 90 degrees $(p<0.05)$. As a result of post-hoc analysis, the angular error value was significantly smaller in the condition of applying the sham taping compared to the dynamic taping and the no taping conditions $(p<0.05)$. There was no significant difference in the comparison according to each condition at 110 degrees $(p>0.05)$. As a result of post-hoc analysis, there was no significant difference in all 3 conditions $(p>0.05)$ (Table 2).

There was a significant difference for each angle under

Table 1. The general characteristics of subjects

$(\mathrm{N}=20)$

\begin{tabular}{lc}
\hline \multicolumn{1}{c}{ Variables } & Values \\
\hline Sex $($ male/female $)$ & $12 / 8$ \\
Age $(\mathrm{y})$ & $23.45(1.53)$ \\
Height $(\mathrm{cm})$ & $168.80(8.93)$ \\
Weight $(\mathrm{kg})$ & $65.25(14.40)$ \\
\hline
\end{tabular}

Values are presented as number only, or mean (SD). 
Table 2. Comparison of shoulder flexion and error values according to taping condition $(\mathrm{N}=20)$

\begin{tabular}{lllrl}
\hline \multicolumn{1}{c}{ Variable } & DT & ST & NT & F \\
\hline Shoulder flexion $\left(^{\circ}\right)$ & & & & \\
50 & $7.62(5.14)^{\mathrm{a}}$ & $8.99(4.16)$ & $10.93(6.04)$ & $4.218^{*}$ \\
90 & $3.35(2.35)^{\mathrm{b}}$ & $2.64(1.86)^{\mathrm{ab}}$ & $4.45(2.31)^{\mathrm{b}}$ & $4.971^{*}$ \\
110 & $3.69(2.03)^{\mathrm{b}}$ & $4.29(3.20)^{\mathrm{b}}$ & $3.82(2.34)^{\mathrm{b}}$ & 0.260 \\
$\mathrm{~F}$ & $8.999^{* *}$ & $15.382^{* *}$ & $20.697^{* *}$ & \\
\hline
\end{tabular}

Values are presented as mean (SD).

DT: dynamic taping, ST: sham taping, NT: no taping.

${ }^{\mathrm{a}}$ Significant difference $(p<0.05)$ from no taping group, ${ }^{\mathrm{b}}$ Significant difference $(p<0.05)$ from shoulder flexion $50^{\circ}$.

${ }^{*} p<0.05,{ }^{*} p<0.01$

the condition of applying the dynamic taping $(p<0.05)$. As a result of post-hoc analysis, the angle error value of 90 degrees and 110 degrees was significantly smaller than that of 50 degrees $(p<0.05)$. There was a significant difference for each angle under the condition of applying the sham taping $(p<0.05)$. As a result of post-hoc analysis, the angle error value of 90 degrees and 110 degrees was significantly smaller than that of 50 degrees $(p<0.05)$. There was a significant difference for each angle in the condition where no tape was applied $(p<0.05)$. As a result of post-hoc analysis, the angle error value of 90 degrees and 110 degrees was significantly smaller than that of 50 degrees $(p<0.05)$ (Table 2).

\section{Discussion}

This study was conducted to find out how dynamic taping affected the joint position sense of shoulder flexion by applying various taping methods to determine the difference in error values of various angles.

Dynamic taping is pre-stretched so that it is ready to provide support immediately when the muscle-tendon unit begins to stretch [11]. Since dynamic taping is applied at 90 degrees of shoulder flexion, the inner range of 50 degrees is the most stimulated. Accordingly, it seems that the application of dynamic taping showed the most significant effect at 50 degrees. For that reason many people with shoulder conditions, such as shoulder impingement syndrome and rotator cuff tear, have pain in the shoulder between 60 and 120 degrees these 2 angles were set: 90 degrees and 120 degrees. Also, these angles were consistent with the angles used to investigate the effect of shoulder height on proprioception from other studies $[15,16]$. Light tactile sensation of the fingertip improves balance on a stationary surface, a small stimulus of less than $1 \mathrm{~N}$ is applied to the finger to reduce body agitation, and the light tactile stimulus promotes the sense of position. It was found that the light tactile sensation had a positive effect on proprioception, skin and joint receptors [17]. At 50 degrees, the direct effect of dynamic taping on the deltoid, skin, joint receptors and muscle spindles obtained through light tactile sensation were more influenced, leading to significant differences [18]. On the other hand, sham taping provided a light tactile sensation to the deltoid rather than direct stimulation of dynamic taping and had a positive effect on proprioception, skin and joint receptors, and it is thought that the most significant difference was obtained at 90 degrees [19].

In previous studies, it was said that applying kinesiology taping did not affect the joint position sense of the shoulder. In addition, it was suggested that kinesiology taping is not an effective treatment method for enhancing joint position sense of the lower extremity as well as the upper extremity [20]. On the other hand, dynamic taping absorbs the load as the tape is stretched in advance and the muscle and skin tissue is stretched and ready to support [21]. Also, when the eccentric exercise ends and the concentric exercise returns, the latent elasticity stored in the tape is applied as an exercise force to help with the muscle load. According to previous papers, dynamic taping can be used for exercise control training to reduce the load on the lower extremity muscles and to improve movement patterns, and it is reported that it improves the quality of movement [22]. In addition, it was reported that motor control using tape could be performed in a way that occupies a part of a load of the upper extremity, and that the ability of muscle tissue is improved by positioning the shoulder [21]. According to other previous papers, it has an effect on static and dynamic balance ability $[23,24]$. As a result of applying dynamic tape on the scapula for shoulder pain, disability, upper body posture, and range of motion in patients undergoing surgery, applying dynamic tape were effective in improving shoulder dysfunction level, 
range of motion and upper extremity posture for patients who underwent acromioplasty and rotator cuff repair [25]. Previous studies suggested that improvement in joint position sense error of about 2 degrees could have a clinical advantage in improving proprioception. Therefore, it is thought that the value of 3 degrees shown in this study will help improve proprioception at 50 degrees of shoulder flexion [26].

The limitations of this study are that the long-term effects are not known because there were no follow-up assessments made after taping, and although there are various methods of applying dynamic taping, the effect of only one method was investigated. In addition, young adults without shoulder injury or disease were studied. Therefore, it is necessary to study the effect of proprioception by applying dynamic taping in the elderly population or individuals with joint instability.

This study was conducted to investigate the effects of somatosensory stimulation and neurophysiological effects between dynamic taping and sham taping on the joint position sense of shoulder in healthy adults in their $20 \mathrm{~s}$. As a result of comparing proprioception according to dynamic taping, sham taping, and no taping in the same shoulder flexion range of motion in subjects with normal shoulder function, the angular error value was significantly smaller in the dynamic taping condition at 50 degrees and sham taping at 90 degrees. The angle error value was significantly smaller. At 110 degrees, there was no significant difference in the taping method. As a result of comparing proprioception sense according to 50 degrees, 90 degrees, and 110 degrees under the same taping condition, the angular errors values were significantly smaller at 90 and 110 degrees for dynamic taping, sham taping and the no taping conditions. It is believed that dynamic taping will help improve joint position sense at 50 degrees of shoulder flexion and there is hope that further studies will be conducted on more subjects and subjects with decreased shoulder proprioception.

\section{Conflicts of Interest}

The authors declared no potential conflicts of interest with respect to the authorship and/or publication of this article.

\section{References}

1. Hertel J. Functional anatomy, pathomechanics, and pathophysi- ology of lateral ankle instability. J Athl Train 2002;37:364-75.

2. Bloem BR, Allum JH, Carpenter MG, Honegger F. Is lower leg proprioception essential for triggering human automatic postural responses? Exp Brain Res 2000;130:375-91.

3. Lee JN, Lim CG. Effects of scapular taping on muscle activity, pain, range of motion and proprioception in subacute stroke patients. J Korea Acad Ind Soc 2013;14:5689-97.

4. Janwantanakul P, Magarey ME, Jones MA, Dansie BR. Variation in shoulder position sense at mid and extreme range of motion. Arch Phys Med Rehabil 2001;82:840-4.

5. McClure P, Tate AR, Kareha S, Irwin D, Zlupko E. A clinical method for identifying scapular dyskinesis, part 1: reliability. J Athl Train 2009;44:160-4.

6. Lee D. Effects of theraband exercise programs on strength, balance and proprioception in elderly. J Korean Soc Integr Med 2014;2:1-8.

7. Shaheen AF, Bull AM, Alexander CM. Rigid and Elastic taping changes scapular kinematics and pain in subjects with shoulder impingement syndrome; an experimental study. J Electromyogr Kinesiol 2015;25:84-92.

8. de la Cruz Torresa B, Albornoz Cabelloa M, Espejo Antúnez L. [Immediate effect of Dynamic Tape ${ }^{\circledR}$ on external torsion tibial clinically painful in a football player. A case report]. Rev Andal Med Deport 2016;9:50-3. Spanish.

9. Kuni B, Mussler J, Kalkum E, Schmitt H, Wolf SI. Effect of kinesiotaping, non-elastic taping and bracing on segmental foot kinematics during drop landing in healthy subjects and subjects with chronic ankle instability. Physiotherapy 2016;102:287-93.

10. Park JT, Kim GD, Han GS. The effects of kinesio taping application on isometric muscular strength of knee joint in each angles of extension, flexion. Korean J Sports Sci 2009;18:1065-74.

11. McNeill W, Pedersen C. Dynamic tape. Is it all about controlling load? J Bodyw Mov Ther 2016;20:179-88.

12. Seo HD, Kim MY, Choi JE, Lim GH, Jung SI, Park SH, et al. Effects of Kinesio taping on joint position sense of the ankle. J Phys Ther Sci 2016;28:1158-60.

13. Huang T, Kim S. The effectiveness of upper limb offload dynamic taping technique on scapular muscles activation during elevation in healthy subjects. Phys Ther Korea 2020;27:93-101.

14. Thelen MD, Dauber JA, Stoneman PD. The clinical efficacy of kinesio tape for shoulder pain: a randomized, double-blinded, clinical trial. J Orthop Sports Phys Ther 2008;38:389-95.

15. Aarseth LM, Suprak DN, Chalmers GR, Lyon L, Dahlquist DT. Kinesio tape and shoulder-joint position sense. J Athl Train 2015;50:785-91.

16. Xiao J, Cui GQ, Wang JQ. Diagnosis of bursal-side partial-thickness rotator cuff tears. Orthop Surg 2010;2:260-5.

17. Jeka JJ, Lackner JR. Fingertip contact influences human postural control. Exp Brain Res 1994;100:495-502.

18. Chapman J, Suprak DN, Karduna AR. Unconstrained shoulder joint position sense does not change with body orientation. J Orthop Res 2009;27:885-90.

19. Lin JJ, Hung CJ, Yang PL. The effects of scapular taping on electromyographic muscle activity and proprioception feedback in healthy shoulders. J Orthop Res 2011;29:53-7.

20. Aytar A, Ozunlu N, Surenkok O, Baltacı G, Oztop P, Karatas M. Initial effects of kinesio ${ }^{\circledR}$ taping in patients with patellofemoral pain syndrome: a randomized, double-blind study. Isokinet 
Exerc Sci 2011;19:135-42.

21. Worsley P, Warner M, Mottram S, Gadola S, Veeger HE, Hermens $\mathrm{H}$, et al. Motor control retraining exercises for shoulder impingement: effects on function, muscle activation, and biomechanics in young adults. J Shoulder Elbow Surg 2013;22: e11-9.

22. Bittencourt N, Leite M, Zuin A, Pereira M, Gonçalves G, Signoretti S. Dynamic taping and high frontal plane knee projection angle in female volleyball athletes. Br J Sports Med 2017; 51:297-8.

23. Lim O, Park S. Comparison of the effects of barefoot, kinesio tape, and dynamic tape on static and dynamic balance in subjects with asymptomatic flexible. Phys Ther Korea 2020;27:78-86.

24. Kwon O, Kim H, Shin W. Comparison of the effects of different types of taping on static and dynamic balance in adults with chronic ankle instability. J Korean Soc Integr Med 2020;8:27-36.

25. Park SJ, Kim SY. The effect of scapular dynamic taping on pain, disability, upper body posture and range of motion in the postoperative shoulder. J Korean Soc Phys Med 2018;13:149-62.

26. Burfeind SM, Chimera N. Randomized control trial investigating the effects of kinesiology tape on shoulder proprioception. J Sport Rehabil 2015;24:405-12. 